\title{
Correlação Genética Entre Perímetro Escrotal e Algumas Características Reprodutivas na Raça Nelore
}

\author{
Evandro Pereira1, Joanir Pereira Eler², José Bento Sterman Ferraz ${ }^{3}$
}

\begin{abstract}
RESUMO - Dados de perímetro escrotal e de características reprodutivas medidas na fêmea foram analisados com o objetivo de estimar o coeficiente de herdabilidade de cada característica e a correlação genética entre as características. Os dados foram obtidos em 11 fazendas pertencentes a uma mesma empresa, situadas nos Estados de São Paulo, Mato Grosso do Sul e Goiás. Os componentes de variância foram estimados por máxima verossimilhança restrita utilizando modelo animal bi-característica. Para as características com medidas repetidas, foram utilizados dois modelos matemáticos: um incluindo ambiente permanente da vaca e outro não. As análises nas quais o modelo não incluía o ambiente permanente da vaca mostraram as seguintes herdabilidades 0,$51 ; 0,12 ; 0,17 ; 0,06$; e 0,13 para perímetro escrotal (PE), idade ao primeiro parto (IPP), dias para o parto (DPP), intervalo de partos (IP) e duração da gestação (DG), respectivamente. Nestas análises, as correlações genéticas foram: -0,22 (PE x IPP), -0,04 (PE x DPP), 0,10 (PE x IP) e -0,04 (PE x DG). Quando o ambiente permanente foi incluído no modelo, as herdabilidades de DPP $(0,07)$ e DG $(0,06)$ foram menores, indicando que modelos sem ambiente permanente podem superestimar a variância genética aditiva dessas duas características. Os parâmetros da característica IP não foram, entretanto, alterados pela inclusão do ambiente permanente no modelo. O PE apresentou correlações genéticas favoráveis com IPP, DPP e DG, mas estas foram geralmente de baixa magnitude. Estes resultados permitem a utilização do perímetro escrotal como critério de seleção para melhorar a eficiência reprodutiva das fêmeas.
\end{abstract}

Palavras-chave: características reprodutivas, correlação genética, modelo animal, perímetro escrotal

\section{Genetic Correlation Between Scrotal Circumference and Some Reproductive Traits in Nellore Cattle}

\begin{abstract}
Data of scrotal circumference and female reproductive traits were analyzed to estimate heritabilities and genetic correlations. Data were obtained from 11 farms by the same company, located in the States of São Paulo, Mato Grosso do Sul and Goiás. Variance components were estimated by restricted maximum likelihood using two-trait animal models. For traits with repeated measures, two mathematical models were used: including or not the permanent environment of the cow. Analyses that not included the permanent environment of the cow showed the following heritabilities $0.51,0.12,0.17,0.06$ and 0.13 for scrotal circumference (SC), age at first calving (AFC), days to calving (DC), calving interval (CI) and gestation length (GL), respectively. In these analyses, the genetic correlations were: -0.22 (SC x AFC), -0.04 (SC x DC), 0.10 (SC x CI) and -0.04 (SC x GL). When permanent environment of the cow were included in the model, heritabilities of DC (0.07) and GL (0.06) were smaller, indicating that models without permanent environment of the cow can overestimate the additive genetic variance of these two traits. However, the CI parameters did not change with the inclusion of permanent environment of the cow in the model. The scrotal circumference showed favorable genetic correlations with AFC, DC and GL, but the values in overall were low. These results allow utilizing SC as a selection criterion to improve the female fertility efficiency.
\end{abstract}

Key Words: reproductive traits, genetic correlation, animal model, scrotal circumference

\section{Introdução}

Os problemas reprodutivos são os principais limitantes da eficiência produtiva em bovinos de corte (MATTOS e ROSA, 1984) e, por isso, a inclusão de características reprodutivas nos objetivos de seleção seria indispensável para a otimização da eficiência econômica do rebanho. Apesar disso, os programas de melhoramento genético mais tradicionais têm dado ênfase quase exclusivamente às características de desempenho ponderal.

O perímetro escrotal (PE) vem sendo utilizado como critério de seleção, por ter correlações genéticas favoráveis com características de sêmen (KNIGHTS et al., 1984), com idade à puberdade em machos e fêmeas (BOURDON e BRINKS, 1986; MARTIN et

\footnotetext{
${ }^{1}$ Mestrando do Curso de Zootecnia (Qualidade e Produtividade Animal) da Fac. de Zootecnia e Engenharia de Alimentos, USP. E.mail: epereira-zoot@yahoo.com

2 Professor Associado da Fac. de Zootecnia e Engenharia de Alimentos, USP, Cx. Postal 23, 13630-970, Pirassununga, SP.E.mail:joapeler@usp.br 3 Professor Titular da Fac. de Zootecnia e Engenharia de Alimentos, USP. E.mail: jbferraz@usp.br
} 
al., 1992; e MOSER et al., 1996) e características de crescimento (KNIGHTS et al., 1984; BOURDON e BRINKS, 1986; KRIESE et al., 1991; LÔBO et al., 1994; e BERGMANN et al., 1996). Autores como MACKINONN et al. (1990) sugeriram que os fatores hormonais responsáveis pelo desenvolvimento testicular inicial nos machos são os mesmos que promovem o desenvolvimento ovariano inicial nas fêmeas. BOURDON e BRINKS (1986) relacionaram as medidas de PE com a fertilidade inerente da fêmea (genes para fertilidade).

BERGMANN et al. (1996) mencionaram vários resultados da literatura, mostrando que o $\mathrm{PE}$ apresenta alta herdabilidade (em média, 50\%) em animais criados em países de clima temperado. Na raça Nelore, os valores obtidos foram semelhantes. ELER et al. (1996) estimaram esta herdabilidade em 52\%. Valores mais baixos foram obtidos por MARTINS FILHO e LÔBO (1991), 0,36 \pm 0,07, e GRESSLER et al. (1998), 0,31 $\pm 0,10$. É necessário salientar que a maioria dos estudos no Brasil considera o PE medido aos 18 meses, enquanto em trabalhos estrangeiros a medida geralmente é feita aos 12 meses (o Bos taurus alcançaria a maturidade sexual antes que o Bos indicus). Facilidade da medição, alta herdabilidade e correlação genética favorável com as características reprodutivas da fêmea aumentam a utilização desta característica em programas de seleção, visando o melhoramento da fertilidade do rebanho.

Com relação às fêmeas, há maior dificuldade de se determinarem características facilmente mensuráveis que sejam geneticamente relacionadas com a fertilidade (JOHNSTON e BUNTER, 1996). Algumas características geralmente utilizadas são idade ao primeiro parto (IPP), intervalo de partos (IP), número de dias para o parto (DPP) e duração da gestação (DG).

A IPP é uma característica de fácil mensuração e reflexo da idade na puberdade, que, por sua vez, pode estar relacionada à velocidade de crescimento da fêmea (PEREIRA et al., 1991).

O IP tem sido utilizado para medir a eficiência reprodutiva, principalmente em gado de leite. A eficácia do sua utilização em gado de corte tem sido questionada, devido ao viés causado pelo adoção de estação de monta fixa, geralmente de curta duração (BOURDON e BRINKS, 1983).

A característica dias para o parto (DPP), calculada como a diferença em dias entre o início da estação de monta e a data do parto para cada estação de monta, seria mais adequada para utilização em gado de corte. Esta característica é função da vari- abilidade das fêmeas em relação à atividade de estro dentro da estação de monta, do número de serviços requeridos e da duração da gestação (NEWNAM et al., 1992). A avaliação de DPP permite que se identifiquem fêmeas com maior fertilidade, ou seja, aquelas que emprenham mais cedo dentro da estação de monta e touros cujas filhas apresentem menor número de dias para o parto. O principal problema na análise de DPP refere-se às vacas que não parem na estação de monta. A maioria dos arquivos históricos não dão conta de modo eficaz desses animais, o que restringe a análise apenas aos animais que pariram, gerando uma amostra de certa forma selecionada. Quando há informações adequadas sobre as fêmeas que pariram e as que não pariram na estação de monta, estas últimas devem ser penalizadas. O BREEDPLAN (Programa Nacional de Registros de Gado de Corte da Austrália) usa em suas análises da raça Angus a penalidade de 21 dias, com base em estudos preliminares de JOHNSTON e BUNTER (1996). As vacas que não parem recebem um valor predito de DPP igual ao maior DPP no grupo contemporâneo mais 21 dias (aproximadamente um desviopadrão da característica e também um ciclo estral).

A DG é outra importante característica relacionada com a reprodução de bovinos, uma vez que vacas com menores períodos de gestação têm vantagem reprodutiva sobre vacas com gestação mais longa (BOURDON e BRINKS, 1983). BOURDON e BRINKS (1982), entretanto, colocam em dúvida a eficácia da utilização da DG como critério de seleção, sugerindo que a seleção para crescimento, associada à seleção para menor duração da gestação, seria menos eficaz que a seleção para maior taxa de crescimento e menor peso ao nascer, pois poderia tanto diminuir a duração da gestação quanto alterar a curva de crescimento. A raça Nelore apresenta, no entanto, média elevada de duração da gestação, cerca de 293 dias (FERRAZ e ELER, 1998), e variabilidade fenotípica.

Considerando a importância das características acima descritas, os objetivos do presente estudo foram: a) estimar os coeficientes de herdabilidade; b) testar a inclusão do ambiente permanente da vaca nos modelos para as características com medidas repetidas (IP, DPP e DG), visando determinar o seu efeito na estimação da herdabilidade; c) estimar as correlações genéticas entre PE, medido aos 18 meses, e características reprodutivas medidas na fêmea (IPP, IP, DPP e DG), com a finalidade de estimar o efeito da seleção para PE sobre a eficiência reprodutiva das fêmeas. 


\section{Material e Métodos}

Foram analisados dados de animais da raça Nelore nascidos entre 1981 e 1997, em 11 fazendas localizadas nos Estados de São Paulo, Mato Grosso do Sul e Goiás, todas pertencentes à Agropecuária CFM Ltda.

As características analisadas e a formação dos grupos contemporâneos são descritas a seguir:

Idade ao primeiro parto (IPP): fazenda (11) + ano (17) + estação de nascimento (2) + grupo de manejo à desmama + fazenda da reprodução + ano da reprodução + estação de monta (2).

Dias para o parto (DPP): calculada como a diferença, em dias, entre o primeiro dia da estação de monta e o dia do parto, em cada estação de monta. $\mathrm{O}$ grupo contemporâneo foi formado por: fazenda da reprodução + ano da reprodução + estação de monta + touro utilizado na reprodução.

A partir da safra de 1997, as vacas que não pariram foram incluídas na análise e penalizadas com 21 dias (aproximadamente um desvio-padrão) a mais do que a vaca que teve o maior número de dias para o parto em seu grupo contemporâneo.

Intervalo de partos (IP): fazenda da reprodução + ano da reprodução + estação de monta + sexo do produto + touro utilizado na reprodução.

Duração da gestação $(D G)$ : fazenda da reprodução + ano da reprodução + estação de monta + sexo do produto + touro utilizado na reprodução.

Perímetro escrotal (PE): fazenda + ano + estação de nascimento + grupo de manejo à desmama + grupo de manejo ao sobreano.

Um resumo descritivo de cada característica é apresentado na Tabela 1.

Para estimação das correlações entre PE e características reprodutivas das fêmeas, foi utilizado o seguinte modelo bi-característica

$$
\left[\begin{array}{l}
y_{1} \\
y_{2}
\end{array}\right]=\left[\begin{array}{cc}
X_{1} & 0 \\
0 & X_{2}
\end{array}\right]\left[\begin{array}{l}
b_{1} \\
b_{2}
\end{array}\right]+\left[\begin{array}{cc}
Z_{1} & 0 \\
0 & Z_{2}
\end{array}\right]\left[\begin{array}{l}
u_{1} \\
u_{2}
\end{array}\right]+\left[\begin{array}{l}
e_{1} \\
e_{2}
\end{array}\right]
$$

$\mathrm{y}_{1}=$ vetor dos registros de produção da característica 1 (IPP, IP, DPP, DG);

$\mathrm{y}_{2}=$ vetor dos registros de produção da característica 2 (PE);

$b_{1}=$ vetor de efeitos fixos para a característica 1 (idade da vaca ao parto, para IP e DG, ou idade da vaca no início da estação de monta, para DPP, como covariável, além do grupo de contemporâneas);

$\mathrm{b}_{2}=$ vetor de efeitos fixos para a característica 2 (idade do animal na data da medição como covariável, além de grupo de contemporâneos);

$\mathrm{u}_{1} \quad=$ vetor de efeitos aleatórios de valor genético para a característica 1 ;

$\mathrm{u}_{2}=$ vetor de efeitos aleatórios de valor genético para a característica 2 ;

$\mathrm{X}_{1}\left(\mathrm{X}_{2}\right)=$ matriz de incidência associando elementos de b1 $\left(\mathrm{b}_{2}\right)$ a $_{\mathrm{y}_{1}}\left(\mathrm{y}_{2}\right)$; e

$\mathrm{Z}_{1}\left(\mathrm{Z}_{2}\right)=$ matriz de incidência associando elementos de $\mathrm{u}_{1}\left(\mathrm{u}_{2}\right)$ a $\mathrm{y}_{1}\left(\mathrm{y}_{2}\right)$.

Para o modelo bi-característica em geral, $\mathrm{E}\left[\mathrm{y}_{\mathrm{i}}\right]=\mathrm{X}_{\mathrm{i}} \mathrm{b}_{\mathrm{i}}$ para $\mathrm{i}=1,2$ e a matriz de variânciacovariância dos elementos aleatórios nomodeloédada por:

$$
V a r\left[\begin{array}{l}
u_{1} \\
u_{2} \\
e_{1} \\
e_{2}
\end{array}\right]=\left[\begin{array}{cccc}
A \sigma_{a 1}^{2} & A \sigma_{a 1 a 2} & 0 & 0 \\
A \sigma_{a 1 a 2} & A \sigma_{a 2}^{2} & 0 & 0 \\
0 & 0 & I \sigma_{e 1}^{2} & 0 \\
0 & 0 & 0 & I \sigma_{e 2}^{2}
\end{array}\right]
$$

em que

A $\quad=$ matriz de parentesco;

$\sigma_{2} \mathrm{a}_{1}, \sigma_{2} \mathrm{a}_{2}=$ variância genética aditiva para as características 1 e 2 , respectivamente;

$\sigma \mathrm{a}_{1} \mathrm{a}_{2}=$ covariância genética aditiva entre características 1 e 2 ; E

$\sigma_{2} \mathrm{e}_{1}, \sigma_{2} \mathrm{e}_{2}=$ variância ambiental para as características 1 e 2, respectivamente.

Para DPP, IP e DG, além do modelo descrito, foi utilizado outro incluindo ambiente permanente da vaca:

$$
\left[\begin{array}{l}
y_{1} \\
y_{2}
\end{array}\right]=\left[\begin{array}{cc}
X_{1} & 0 \\
0 & X_{2}
\end{array}\right]\left[\begin{array}{l}
b_{1} \\
b_{2}
\end{array}\right]+\left[\begin{array}{cc}
Z_{1} & 0 \\
0 & Z_{2}
\end{array}\right]\left[\begin{array}{l}
u_{1} \\
u_{2}
\end{array}\right]+W_{1} p_{1}+\left[\begin{array}{l}
e_{1} \\
e_{2}
\end{array}\right]
$$

em que $\mathrm{p}_{1}$ é o vetor de ambiente permanente e efeitos genéticos não aditivos da vaca e $\mathrm{W}_{1}$, a matriz que associa $\mathrm{p}$ com y. Os demais fatores são como definidos anteriormente.

Os componentes de variância foram obtidos por máxima verossimilhança restrita, utilizando-se o programa computacional MTDFREML - Multiple Trait Derivative Free Restricted Maximum Likelihood (BOLDMAN et al., 1993).

Considerou-se que o critério de convergência tinha sido alcançado, quando a variância dos valores do logaritmo da função de verossimilhança era igual ou menor que $10^{-9}$. Como há possibilidade de se obterem máximos locais, em vez do máximo global (PRES et al., 1986), várias reinicializações foram executadas no sentido de se assegurar a convergência no máximo global da função de verossimilhança. 
PEREIRA et al.

Tabela 1 - Número de observações $(N)$, média $(\bar{X})$, desvio-padrão (DP), coeficiente de variação (CV), valores mínimo e máximo e número de grupos contemporâneos (NGC) para cada característica reprodutiva analisada

Table 1 - Number of observations $(\mathrm{N})$, average $(\overline{\mathrm{X}})$, standard deviation $(\mathrm{SD})$, coefficient of variation $(\mathrm{CV})$, minimum and maximum values and number of contemporary groups (NCG) for each analyzed reproductive trait

\begin{tabular}{|c|c|c|c|c|c|c|c|}
\hline $\begin{array}{l}\text { Característica }^{1} \\
\text { Trait }^{1}\end{array}$ & $\mathrm{~N}$ & $\bar{X}$ & $\begin{array}{l}\text { DP } \\
S D\end{array}$ & $\mathrm{CV}(\%)$ & $\begin{array}{l}\text { Valor mínimo } \\
\text { Minimum value }\end{array}$ & $\begin{array}{l}\text { Valor máximo } \\
\text { Maximum value }\end{array}$ & $\begin{array}{l}\text { NGC } \\
N C G\end{array}$ \\
\hline $\begin{array}{l}\mathrm{PE} \\
\text { SC } \\
\text { IPP }\end{array}$ & 16.999 & 28,3 & 3,2 & 11,4 & 12 & 44 & 700 \\
\hline $\begin{array}{l}\text { AFC } \\
\text { DPP }\end{array}$ & 8.538 & 1070,1 & 104,2 & 9,7 & 688 & 1475 & 600 \\
\hline $\begin{array}{l}\text { DC } \\
\text { IP }\end{array}$ & 15.669 & 323,5 & 25,5 & 7,9 & 260 & 400 & 175 \\
\hline $\begin{array}{l}\text { CI } \\
\text { DG }\end{array}$ & 9.476 & 394,1 & 64,0 & 16,2 & 301 & 650 & 122 \\
\hline G & 4.391 & 295,2 & 7,5 & 2,5 & 260 & 320 & 52 \\
\hline
\end{tabular}

\section{Resultados e Discussão}

Estimativas de componentes de (co)variância são mostradas na Tabela 2. Observou-se que a inclusão do ambiente permanente da vaca diminuiu o componente de variância genética aditiva direta de DPP e DG. A utilização dos dois modelos, com e sem ambiente permanente da vaca, mostrou que grande parte da variância tida como genética aditiva é, na verdade, derivada dos efeitos genéticos não-aditivos e da diferença de ambiente permanente das vacas. Portanto, se este efeito não for incluído no modelo matemático, a variância genética pode ser superestimada.

Para a característica intervalo de partos, a inclusão do ambiente permanente alterou os componentes de variância. Isso indica que a variância decorrente do ambiente permanente da vaca contribui pouco para a variância fenotípica da característica.

Os parâmetros genéticos são apresentados na Tabela 3. Para perímetro escrotal, o valor obtido para o coeficiente de herdabilidade $\left(\mathrm{h}^{2}\right)$ foi próximo do valor médio $(0,45)$ citado por KOOTS et al. (1994), em uma revisão de trabalhos envolvendo várias raças em vários países. ELER et al. (1996) encontraram h ${ }^{2}$ igual a 0,52 , trabalhando com a raça Nelore. Outros estudos com a raça Nelore comprovaram a alta herdabilidade desta característica (LÔBO et al., 1994; BERGMANN et al., 1996; PEÑA et al., 1998). GRESSLER et al. (1998) obtiveram $\mathrm{h}^{2}$ igual a $0,31 \pm 0,10$, mas o número de observações utilizado na análise foi pequeno (607).
Os coeficientes de herdabilidade obtidos para características reprodutivas das fêmeas foram baixos. TOELLE e ROBISON (1985) já discutiram a baixa magnitude do componente de variância genético para estas características. Para IPP, obteve-se $\mathrm{h}^{2}$ igual a 0,12 . Os resultados encontrados na literatura científica variaram de 0,02 a 0,46 (PIMENTA FILHO e LEITE, 1989; PEREIRA et al., 1991; ABREU et al., 1991; MARTINS FILHO et al., 1991; PÁDUA et al., 1994; MERCADANTE, 1995; e GRESSLER, 1998). KOOTS et al. (1994), em sua revisão, obtiveram média igual a 0,14 . A idade ao primeiro parto é, no entanto, função da idade à primeira exposição ao touro ou primeira inseminação. Na verdade, pode-se considerar mais de uma característica para IPP. Por exemplo: IPP14 para os animais expostos pela primeira vez aos 14 meses e IPP24 para os animais expostos pela primeira vez aos 24 meses. Estas análises estão sendo processadas e os resultados serão publicados em outro trabalho.

O número de dias para o parto (DPP) apresentou-se como importante característica na avaliação da fertilidade das fêmeas, avaliada em todas as categorias de vacas $(14,18,24$ e 36 meses e adultas), inclusive ajustando para idade no início da estação de monta, dentro de cada categoria. Isto permite a utilização de grande volume de dados. Os resultados mostraram, no entanto, baixa herdabilidade $(0,07$ na análise incluindo os efeitos de ambiente permanente da vaca e 0,17 na análise sem ambiente permanente). Valores similares são encontrados na literatura. MEYER et 
Rev. bras. zootec.

Tabela 2 - Componentes de variância e covariância para características reprodutivas na raça Nelore, com modelo incluindo ou não ambiente permanente da vaca

Table 2 - Variance and covariance components for reproductive traits in Nelore cattle, with models including or not permanent environment of cow

Componentes de (co)variância ${ }^{1}$

(Co)Variance components ${ }^{1}$

Modelo sem amb. perm.

Model without permanent environment

$\sigma^{2}{ }_{\mathrm{g}}$
$\sigma^{2}{ }^{\mathrm{e}}$
$\sigma^{2}{ }_{\mathrm{t}}$

$\sigma^{2}$
$\sigma_{\mathrm{g} 1 \mathrm{~g} 2}$

$\sigma_{\mathrm{e} 1 \mathrm{e} 2}$

$\sigma_{\mathrm{t} 1 \mathrm{t} 2}$

Modelo com ambiente permanente

Model with permanent environment

\begin{tabular}{lllrrr}
$\sigma^{2} \mathrm{~g}$ & 3,34 & - & 29,83 & 157,30 & 3,26 \\
$\sigma^{2}$ & - & - & 38,14 & 0,00 & 4,32 \\
$\sigma^{2}$ & 3,27 & - & 330,80 & 2640,48 & 42,85 \\
$\sigma^{2}$ & 6,61 & - & 398,77 & 2794,78 & 50,43 \\
$\sigma_{\mathrm{g} 1 \mathrm{~g} 2}$ & - & - & $-0,57$ & 2,29 & $-0,16$ \\
$\sigma_{\mathrm{e} 1 \mathrm{e} 2}$ & - & - & 0,00 & 0,00 & 0,00 \\
$\sigma_{\mathrm{t} 1 \mathrm{t} 2}$ & - & - & $-0,57$ & 2,29 & $-0,16$ \\
\hline
\end{tabular}

${ }^{1} \sigma_{g}^{2}=$ variância genética aditiva direta, $\sigma_{p}^{2}=$ variância de ambiente permanente, $\sigma^{2}{ }_{e}=$ variância ambiental, $\sigma_{t}^{2^{g}}=$ variância fenotípica, $\sigma_{\mathrm{g} 1 \mathrm{~g} 2}=$ covariância entre os efeitos genéticos de PE (índice 1) e características reprodutivas das fêmeas (índice 2 ), $\sigma_{\mathrm{e} 1 \mathrm{e} 2}=$ covariância entre os efeitos ambientais de 1 e 2, $\sigma_{\mathrm{t} 1 \mathrm{t} 2}=$ covariância fenotípica entre 1 e 2.

$2 \mathrm{PE}=$ perímetro escrotal (em $\mathrm{cm}$ ); IPP = idade ao primeiro parto (em dias); DPP = dias para o parto (em dias); $\mathrm{IP}=$ intervalo de partos (em dias); e DG = duração da gestação (em dias).

${ }_{1}^{1} \sigma_{g}{ }_{g}=$ direct additive genetic variance, $\sigma_{p}^{2}=$ permanent environmental variance, $\sigma^{2}{ }_{e}=$ environmental variance, $\sigma_{t}^{2}=$ phenotypic variance, $\sigma_{g 1 g 2}=$ genetic covariance between trait 1 (SC) and trait 2 (female reproductive traits), $\sigma_{e 1 e 2}=$ environmental covariance between 1 and 2, $\sigma_{t 112}=$ phenotypic covariance between 1 and 2 .

$2 S C=$ scrotal circumference (given in cm); $A F C$ = age at first calving (in days); $D C=$ days to calving (in days); $C l=$ calving interval (in days); and $G L=$ gestation length (in days).

Tabela 3 - Parâmetros genéticos e de ambiente para características reprodutivas na raça Nelore em modelos com ou sem ambiente permanente da vaca

Table 3 - Genetic and environmental parameters for reproductive traits in Nellore cattle in models with or without permanent environment of cow

\begin{tabular}{llcccl}
\hline & \multicolumn{5}{c}{ Características $^{2}$} \\
\cline { 2 - 6 } Parâmetros $^{1}$ & \multicolumn{5}{c}{ Trait $^{2}$} \\
Parameter $^{1}$ & $\mathrm{PE}$ & $\mathrm{IPP}$ & $\mathrm{DPP}^{3}$ & $\mathrm{IP}^{3}$ & $\mathrm{DG}^{3}$ \\
\hline $\mathrm{h}^{2}$ & $\mathrm{SC}$ & $\mathrm{AFC}$ & $D C^{3}$ & $C I^{3}$ & $G^{3}$ \\
$\mathrm{c}^{2}$ & $0,51(0,51)$ & 0,12 & $0,17(0,07)$ & $0,06(0,06)$ & $0,13(0,06)$ \\
$\mathrm{e}^{2}$ & - & - & 0,10 & 0,00 & 0,09 \\
$\mathrm{r}_{\mathrm{g}}$ & $0,49(0,49)$ & 0,88 & $0,83(0,83)$ & $0,94(0,94)$ & $0,87(0,85)$ \\
\hline
\end{tabular}

$1 \mathrm{~h}^{2}=$ estimativa do coeficiente de herdabilidade para efeitos genéticos diretos; $\mathrm{c}^{2}=$ fração da variância fenotípica devida ao ambiente permanente; $\mathrm{e}^{2}$ = fração da variância fenotípica devida ao ambiente temporário; $\mathrm{r}_{\mathrm{g}}=$ correlação genética entre PE e características reprodutivas das fêmeas.

$2 \mathrm{PE}$ = perímetro escrotal; IPP = idade ao primeiro parto; $D P P=$ dias para o parto; IP = intervalo de partos; e DG = duração da gestação.

3 Valores entre parênteses são referentes às análises com ambiente permanente.

$1 h^{2}=$ estimate of heritability for direct genetic effects; $c^{2}=$ fraction of phenotypic variance due to permanent environment; $e^{2}=$ fraction of phenotypic variance due to temporary environment; $r_{g}=$ correlation between $S C$ and female reproductive traits.

$2 S C=$ scrotal circumference; $A F C=$ age at first calving; $D C=$ days to calving; $C l=$ calving interval; and $G L=g e s t a t i o n ~ l e n g t h$.

3 Values between parenthesis refer to analyses with permanent environment. 
al. (1990) obtiveram 0,05, 0,08 e 0,09 para as raças Hereford, Angus e zebuínos cruzados, respectivamente. Em outra análise, MEYER et al. (1991) obtiveram valores de 0,07 a 0,13 para as mesmas raças. Mais recentemente, JOHNSTON e BUNTER (1996) encontraram herdabilidade de 0,07 para DPP. Todos esses autores incluíram o ambiente permanente da vaca no modelo. No Brasil, GRESSLER et al. (1998) encontraram, na raça Nelore, $h^{2}$ igual a 0,11 $\pm 0,05$ e $0,07 \pm 0,08$ para data do primeiro parto e data do segundo parto, respectivamente, na raça Nelore. A data do parto é uma característica similar à DPP.

Para intervalo de partos, KOOTS et al. (1994) observaram herdabilidade média de 0,10 em trabalhos com várias raças, valor similar ao encontrado no presente estudo.

A duração da gestação apresentou valores de herdabilidade abaixo dos relatados em vários estudos no Brasil e no exterior. No presente trabalho, a DG foi analisada como característica da vaca. SCARPATI et al. (1998), estudando a DG como característica do bezerro na raça Nelore, encontraram valores de 0,58 e 0,49 para modelos que incluíam ou não ambiente permanente da vaca. Outros valores encontrados na literatura variam de $0,30 \pm 0,18$ a 0,48 (BURFENING et al., 1978; BOURDON e BRINKS, 1982; MACNEIL et al., 1984; AZZAM e NIELSEN, 1987; e McGUIRK et al., 1998). ALENCAR et al. (1998), usando método dos quadrados mínimos, obtiveram coeficientes de herdabilidade de $0,17 \pm 0,14$ para DG de vacas Nelore e $0,69 \pm 0,31$ para vacas $1 / 2$ Canchim $1 / 2$ Nelore, considerando a DG como característica do bezerro.

O componente $\mathrm{c}^{2}$ representa a fração da variância fenotípica decorrente dos efeitos de ambiente permanente da vaca e efeitos genéticos não-aditivos. Pode-se observar na Tabela 3 que este componente contribuiu com 10 e 9\% da variância fenotípica para as características DPP e DG, respectivamente. SCARPATI et al. (1998) relataram contribuição de $5 \%$ do ambiente permanente na variância total da DG, enquanto MEYER et al. (1991) observaram contribuições de 16,6 ; 3,5; e 3,4\% para DPP nas raças Hereford, Angus e zebuínos cruzados, respectivamente. Nas análises em que o modelo não incluíu os efeitos de ambiente permanente, os coeficientes de herdabilidade para DPP e DG foram superestimados. O mesmo não se aplica a IP, em que inclusão do ambiente permanente não alterou a estimativa de herdabilidade. Como mencionado anteriormente, a característica IP é muito influenciada pela existência de estação de monta fixa em gado de corte, além de sofrer a influência de fatores que atuam sobre o parto atual e o anterior.

As correlações entre PE e as características reprodutivas obtidas neste estudo foram, em geral, favoráveis, embora de baixa magnitude, exceto para intervalo de partos. A correlação genética $\left(r_{g}\right)$ favorável entre PE e IPP $(-0,22)$ está de acordo com a literatura. TOELLE e ROBISON (1985) obtiveram $\mathrm{r}_{\mathrm{g}}$ igual a -0,14 na raça Hereford, em análise de meioirmãos paternos, enquanto a correlação obtida por MARTINS FILHO e LÔBO (1991) foi igual a -0,44.

Para a correlação entre PE e DPP, os valores obtidos foram $-0,06$ e $-0,04$, respectivamente, nas análise com e sem ambiente permanente da vaca. MEYER et al. (1991) obtiveram valores superiores $(-0,25,-0,28$ e - $-0,41$ nas raças Hereford, Angus e zebuínos cruzados). Os valores obtidos por MORRIS e CULLEN (1994) para a correlação entre PE e data do parto, em taurinos, variaram de $-0,09 \pm 0,45$ a $0,08 \pm 0,55$. Na raça Nelore, GRESSLER et al. (1998) encontraram correlações genéticas de 0,21 e - $-0,35$ entre $\mathrm{PE}$ e as datas do primeiro e segundo partos, respectivamente, em uma análise com pequeno número de observações.

Para PE x DG, os valores obtidos foram $-0,05$ e $-0,04$ nas análise com e sem ambiente permanente. Poucos são os resultados encontrados na literatura. KNIGHTS et al. (1984), BOURDON e BRINKS (1986), KRIESE et al. (1991), LÔBO et al.(1994) e BERGMANN et al.(1996) obtiveram correlação positiva entre PE e características de crescimento e BOURDON e BRINKS (1982), correlação negativa entre DG e características de crescimento, exceto para peso ao nascer. Assim, esperar-se-ia que o PE fosse negativamente correlacionado com a DG, como foi encontrado na presente análise. Entretanto, a correlação obtida nesta pesquisa foi baixa magnitude.

A correlação entre PE e IP observada neste estudo foi positiva $(0,10)$, o que difere de outros trabalhos que relatam correlação negativa (TOELLE e ROBISON, 1985; KOOTS et al., 1994; e GRESSLER et al., 1998).

\section{Conclusões}

As correlações genéticas favoráveis entre PE aos 18 meses de idade e características reprodutivas das fêmeas (com exceção de IP) permitem a utilização do perímetro escrotal como critério de seleção para melhorar a eficiência reprodutiva das fêmeas. Entretanto, devido a sua baixa magnitude, outras características reprodutivas, mais objetivas, devem 
ser investigadas, como a taxa de prenhez em novilhas e taxa de permanência (stayability) nas vacas (SNELLING, 1994; SNELLING et al., 1995).

A não-inclusão do ambiente permanente da vaca nos modelos para DPP e DG pode levar à superestimação das estimativas de herdabilidade.

\section{Agradecimento}

À Agropecuária CFM, pela cessão do conjunto de dados; ao CNPq e à FAPESP, pelo apoio financeiro; e à Tecnóloga em processamento de dados Elisângela Chicaroni de Mattos, pela ajuda na organização dos dados.

\section{Referências Bibilográficas}

ABREU, U.G.P., FILHO, E.B.O., SOBRINHO, E.B. Herança da precocidade sexual em novilhas Nelore, variedade pele rosa. In: REUNIÃO ANUAL DA SOCIEDADE BRASILEIRA DE ZOOTECNIA, 28, João Pessoa, 1991. Anais... João Pessoa: SBZ, 1991. p.551.

ALENCAR, M.M., BARBOSA, R.T., NOVAES, A.P. Características produtivas de fêmeas da raça Nelore e cruzadas $1 / 2$ Canchim+ $1 / 2$ Nelore. In: REUNIÃO ANUAL DA SOCIEDADE BRASILEIRA DEZOOTECNIA, 35, Botucatu, 1998. Anais... Botucatu: SBZ, 1998. v.3, p. 511-513.

AZZAM, S.M., NIELSEN, M.K. 1987. Genetic parameters for gestation length, birth date and first breeding date in beef cattle. J. Anim. Sci., 64: 348-56.

BERGMANN, J.A.G., ZAMBORLINI, L.C., PROCÓPIO, C.S.O. et al. 1996. Estimativas de parâmetros genéticos do perímetro escrotal e do peso corporal em animais da raça Nelore. Arq. Bras. Med. Vet. Zootec., 48: 69-78.

BOLDMAN, K.G., KRIESE, L.A., VAN VLECK, L.D. et al. A manual for use of MTDFREML: a set of programs to obtain estimates of variances and covariances. USDA-ARS, 1993. $120 \mathrm{p}$.

BOURDON, R.M., BRINKS, J.S. 1982. Genetic, environmental and phenotypic relationships among gestation length, birth weight, growth traits and age at first calving in beef cattle. J. Anim. Sci., 55(3):542-53.

BOURDON, R.M., BRINKS, J.S. 1983. Calving date versus calving interval as a reproductive measure in beef cattle. J. Anim. Sci., 57(6):1412-417.

BOURDON, R.M., BRINKS, J.S. 1986. Scrotal circumference in yearling Hereford bulls: adjustment factors, heritabilities and genetic, environmental and phenotypic relationships with growth traits. J. Anim. Sci., 62:958-67.

BURFENING, P.J., KRESS, D.D., FRIEDRICH, R.L. et al. 1978. Phenotypic and genetic relationships between calving ease, gestation length, birth weight and preweaning growth. J. Anim. Sci., 47:595.

ELER, J.P., FERRAZ, J.B.S., SILVA, P.R. 1996. Parâmetros genéticos para peso, avaliação visual e circunferência escrotal na raça Nelore, estimados por modelo animal. Arq. Bras. Med. Vet. Zootec., 48(2):203-13.

FERRAZ, J.B.S., ELER, J.P. 1998. Sumário de touros Nelore, São José do Rio Preto: CFM - Agropecuária CFM Ltda. p.10.

GRESSLER, S.L. Estudo de fatores de ambiente e parâmetros genéticos de algumas características reprodutivas em animais da raça Nelore. UFMG: Belo Horizonte, 1998. 149p. Dissertação (Mestrado) - Escola de Veterinária, Universidade Federal de Minas Gerais, 1998.

GRESSLER, S.L., BERGMANN, J.A.G., PENNA, V.M. et al. Estudo das associações genéticas entre perímetro escrotal e características reprodutivas de fêmeas da raça Nelore. In: REUNIÃO ANUAL DA SOCIEDADE BRASILEIRA DE ZOOTECNIA, 35, Botucatu, 1998. Anais... Botucatu: SBZ, 1998. v.3, p.368-370.

JOHNSTON, D.J., BUNTER, K.L. 1996. Days to calving in Angus cattle: genetic and environmental effects, and covariances with other traits. Livest. Prod. Sci., 45:13-22.

KNIGHTS, S.A., BAKER, R.L., GIANOLA, D. et al. 1984. Estimates of heritabilities and of genetic and phenotypic correlations among growth and reproductive traits in yearling Angus bulls. J. Anim. Sci., 58(4):887-93.

KOOTS, K.R., GIBSON, J.P., SMITH, C. et al. 1994. Analyses of published genetic parameter estimates for beef production traits: 1. Heritability. Anim. Breeding Abstr., 62(5):309-38.

KRIESE, L.A., BERTRAND, J.K., BENYSHEK, L.L. 1991. Age adjustment factors, heritabilities and genetic correlations for scrotal circumference and related growth traits in Hereford and Brangus bulls. J. Anim. Sci., 69:478-89.

LÔBO, R.B., REYES, A., FERRAZ, J.B.S. et al. Bivariate animal model analysis of growth weights and scrotal circumference of Nellore cattle in Brazil. In: WORLD CONGRESS ON GENETICS APPLIED TO LIVESTOCK PRODUCTION, 5, Guelph, Canada, 1994. Proceedings... Guelph, Canada, 1994. p.199-202.

MACKINNON, M.J., TAYLOR, J.F., HETZEL, D.J.S. 1990. Genetic variation and covariation in beef cow and bull fertility. J. Anim. Sci., 68(5):1208-214.

MACNEIL, M.D., CUNDIFF, L.V., DINKEL, C.A. et al. 1984. Genetic correlations among sex-limited traits in beef cattle. J. Anim. Sci., 58(5):1171-180.

MARTIN, L.C., BRINKS, J.S., BOURDON, R.M. et al. 1992. Genetic effects on beef heifer puberty and subsequent reproduction. J. Anim. Sci., 70:4006-4017.

MARTINS FILHO, R., LÔBO, R.B. 1991. Estimates of genetic correlations between sire scrotal circumference and offspring age at first calving in Nellore cattle. Rev. Bras. Genet., 14(1):209-12.

MARTINS FILHO, R., LÔBO, R.B., DA SILVA, P.R. Efeitos genéticos e de meio sobre características reprodutivas de fêmeas da raça Nelore. In: REUNIÃO ANUAL DA SOCIEDADE BRASILEIRA DE ZOOTECNIA, 28, João Pessoa, 1991. Anais... João Pessoa: SBZ, 1991. p.572.

MATTOS, S., ROSA, A.N. 1984. Desempenho reprodutivo de fêmeas de raças zebuínas. Inf. Agropec., 10:29.

McGUIRK, B.J., GOING, I., GILMOUR, A.R. 1998. The genetic evaluation of beef sires used for crossing with dairy cows in the UK: 2. Genetic parameters and sire merit predictions for calving survey traits. Anim. Sci., 66:47-54.

MERCADANTE, M.E.Z. Estudo das relações genéticoquantitativas entre características de reprodução, crescimento e produção em fêmeas da raça Nelore. Ribeirão Preto, 1995. 90p. Dissertação (Mestrado) - Faculdade de Medicina de Ribeirão Preto, Universidade de São Paulo, 1995.

MEYER, K., HAMMOND, K., MACKINNON, M.J. et al 1991. Estimates of covariances between reproduction and growth in australian beef cattle. J. Anim. Sci., 69:3533-543.

MEYER, K., HAMMOND, K., PARNELL, P.F. et al. 1990 Estimates of heritability and repeatability for reproductive traits in australian beef cattle. Livest. Prod. Sci., 25:15-30. 
MORRIS, C.A., CULLEN, N.G. 1994. A note on genetic correlation between pubertal traits of males or females and lifetime pregnancy rate in beef cattle. Livest. Prod. Sci., 39:291-297.

MOSER, D.W., BERTRAND, J.K., BENYSHEK, L.L. et al. 1996. Effects of selection for scrotal circumference in Limousin bulls on reproductive and growth traits of progeny. J. Anim. Sci., 74:2052-2057.

NEWMAN, S., MORRIS, C.A., BAKER, R.L. et al. 1992. Genetic improvement of beef cattle in New Zealand: breeding objectives. Livest. Prod. Sci., 32:111-30.

PADUA, J.T., MUNARI, D.P., WATANABE, Y.F. et al. 1994. Avaliação de efeitos de ambiente e da repetibilidade de características reprodutivas em bovinos da raça Nelore. R. Soc. Bras. Zootec., 23(1):126-32.

PEÑA, C.D.O., CAMPOS, F.P., FEITOSA, J.V. et al. Estimativas de herdabilidade do perímetro escrotal, peso corporal e suas relações em tourinhos da raça Nelore no Paraguai. In: REUNIÃO ANUAL DA SOCIEDADE BRASILEIRA DEZOOTECNIA, 35, Botucatu, 1998. Anais... Botucatu: SBZ, 1998. v.3, p.365-367.

PEREIRA, J.C.C., AYALA, J.M.N., OLIVEIRA, H.N. 1991. Efeitos genéticos e não-genéticos sobre a idade ao primeiro parto e o intervalo entre partos de duas populações da raça Nelore. Arq. Bras. Med. Vet. Zootec., 42:93-102.

PIMENTA FILHO, E.C., LEITE, M.E.V.F. Idade à primeira cria e intervalo entre partos em vacas Nelore. In: REUNIÃO ANUAL DA SOCIEDADE BRASILEIRA DE ZOOTECNIA, 26, Porto Alegre, 1989. Anais... Porto Alegre: SBZ, 1989. p.252.
PRES, W.H., FLANNERY, B.P., TEUKOLSKY, S.A. et al. Numerical recipes. Cambridge: Cambridge University Press, 1986. 818p.

SCARPATI, M.T.V., LÔBO, R.B., REYES, A. et al. Modelos animais alternativos para estimação de parâmetros genéticos e fenotípicos do período de gestação na raça Nelore. In: REUNIÃO ANUAL DA SOCIEDADE BRASILEIRA DE ZOOTECNIA, 35, Botucatu, 1998. Anais... Botucatu: SBZ, 1998. v.3, p.452-454.

SNELLING, W.M. Genetic analyses of binary stayability measures of beef females. Colorado State University, Fort Collins, Co, Tese PhD, 1994. 174p.

SNELLING, W.M., GOLDEN, B.L., BOURDON, R.M. 1995. Within-herd genetic analyses of stayability of beef females. J. Anim. Sci., 73:993-1001.

TOELLE, V.D., ROBISON, O.W. 1985. Estimates of genetic correlations between testicular measurements and female reproductive traits in cattle. J. Anim. Sci., 60(1):89-100.

Recebido em: 07/12/98

Aceito em: 25/04/00 